\title{
SABERES LOCALES Y FRUTOS COMESTIBLES DE PLANTAS NATIVAS EN LA COMARCA ANDINA DEL PARALELO 42 ${ }^{\circ}$ (PATAGONIA, ARGENTINA)
}

\author{
LOCAL KNOWLEDGE AND EDIBLE FRUITS OF NATIVE PLANTS IN THE ANDEAN \\ REGION OF THE PARALLEL $42^{\circ}$ (PATAGONIA, ARGENTINA)
}

\footnotetext{
${ }^{1 *}$ Juan José OCHOA, ${ }^{2}$ Ezequiel Luis Nehuén MONCUNILL, 1,3 Javier Guido PUNTIERI, ${ }^{2}$ Brisa Serena GÜENULEO, ${ }^{4}$ Sonia Edith STEFE, ${ }^{1}$ María Luz CARDOZO, ${ }^{2}$ Francisco NERANZI BARRIGA, ${ }^{1}$ Eduardo Enrique MARTINEZ, ${ }^{2}$ Sergio TORREGO, ${ }^{1}$ Santiago NAON

${ }^{1}$ Instituto de Investigaciones en Recursos Naturales, Agroecología y Desarrollo Rural (Universidad Nacional de Río Negro - Consejo Nacional de Investigaciones Científicas y Técnicas, CONICET, Argentina). ${ }^{2}$ Estudiantes de Licenciatura en Agroecología (Universidad Nacional de Río Negro). ${ }^{3}$ CONICET (Argentina). ${ }^{4}$ Estudiante de la Tecnicatura en Viveros. ${ }^{*}$ jjochoa@unrn.edu.ar
}

\section{RESUMEN}

En los bosques andinos de la región conocida como Comarca Andina del Paralelo $42^{\circ}\left(\mathrm{CAP} 42^{\circ}\right)$, los frutos silvestres de plantas nativas han sido utilizados desde tiempos históricos; sin embargo, no existen estudios que caractericen el aprovechamiento de estas especies en el área. Este trabajo es una aproximación etnobotánica para documentar los saberes y prácticas de aprovechamiento locales sobre este tipo de recursos nativos. Entrevistamos (entrevistas semi-estructurada, abierta y observación participante) a 22 pobladores de dos parajes rurales de la CAP $42^{\circ}$, en íntimo contacto con áreas boscosas: El Desemboque (El Hoyo) y Río Turbio (Parque Nacional Lago Puelo, Parque Provincial y Reserva Forestal Río Turbio). Documentamos una riqueza de 12 especies silvestres nativas con frutos comestibles y tres especies exóticas. Las especies nativas con mayor consenso de mención fueron el maqui, Aristotelia chilensis, el calafate, Berberis microphylla, y la murta, Ugni molinae. Todas las especies nativas muestran cierto grado de desuso respecto a generaciones pasadas, tanto en términos de riqueza de especies como en lo que respecta a la multiplicidad de usos. Factores ecológicos y transformaciones sociales explicarían dichos cambios en el uso. El maqui es la única especie nativa actualmente comercializada, aunque en la zona de estudio su aprovechamiento comercial no parece ser una práctica extendida. Los pobladores entrevistados reconocen la demanda de este recurso por el mercado nacional e internacional. Se sugiere llevar adelante: a) investigaciones etnobotánicas en diferentes áreas de la CAP $42^{\circ}$ que evalúen las valoraciones locales sobre el maqui y el comercio asociado a este recurso; b) estudios ecológicos del impacto de la recolección en las poblaciones silvestres de las especies nativas, particularmente sobre Aristotelia chilensis; y c) ensayos de selección y cultivo de las especies nativas valoradas y demandadas por el mercado.

Palabras-clave: Aristotelia chilensis, cambios de uso, comercialización, recolección, recursos silvestres

\section{ABSTRACT}

The "Comarca Andina del Paralelo 42" (Andean Country of the Parallel $42^{\circ}-\mathrm{CAP} 42^{\circ}$ ) is located in the Andean Patagonia Forest Matrix where native edible fruits were used from historical times there are not studies in this regard. In this first ethnobotanical approach, we document local knowledge and practice about this wild resource. We interviewed (semi-structured interviews, open and participant observation) to 22 rural settlers of two areas in CAP $42^{\circ}$ : El Desemboque (El Hoyo) y Río Turbio (Parque Nacional Lago Puelo, Parque Provincial y Reserva Forestal Río Turbio). We recorded 12 native and three exotic wild plants with edible fruits. Aristotelia chilensis (maqui), Berberis microphylla (calafate) y Ugni molinae (murta) were the native species with the highest number of citation. Taken into account used species richness and use multiplicity of each in past and present times all the native species are less used than in past generation. Social and ecological transformation would explain this use change on native flora. The "maqui" is the only native species currently marketed by interviewed. Nevertheless in the study area its commercial use does not seem to be an extended practice. The interviewed recognize the maqui`s recent demand by national and international market. We suggest: a) carrying out ethnobotanical assessments in different areas of the CAP $42^{\circ}$ to evaluate the "maqui" local valuation and the market associated with this resource; b) ecological research to assessment the impact of harvesting on the dynamic and production of wild usefull species (specially for maqui); and c) selection and cultivation trials for species valued and demanded by the market.

Keywords: Aristotelia chilensis, change in use, commercialization, harvest, wild resources 


\section{INTRODUCCIÓN}

Los frutos silvestres constituyen un recurso forestal no maderable (PFNM) de gran importancia para poblaciones rurales alrededor del mundo (PIMENTEL et al., 1997; BHARUEHA y PRETTY, 2010). En los Bosques Andinos de la Patagonia Argentina-Chilena, este tipo de PFNM ha tenido importancia en la alimentación indígena desde tiempos prehistóricos (BERNAL et al., 2007) e históricos (CIAMPAGNA y CAPPARELLI, 2012), y más recientemente de las poblaciones rurales indígenas-criollas (LADIO, 2001; OCHOA et al., 2010). Tratándose de una región de clima templadofrío, donde los recursos vegetales silvestres comestibles ocurren de manera estacional, la contribución de los frutos silvestres (carbohidratos, minerales y compuestos antioxidantes) resulta en un aporte clave a las dietas de subsistencia de las poblaciones rurales.

En la actualidad, las prácticas históricas de uso de la biodiversidad se ven re-significadas en los contextos de mercado, en los cuales los recursos silvestres, antaño anclados a prácticas culturales de recolección - intercambio - consumo doméstico, comienzan a ser parte de circuitos de recolección - venta - consumo fuera de los territorios de recolección. Estas transformaciones generan nuevas presiones sobre los recursos, por ejemplo, sobre-explotación, pero también representan oportunidades para la revalorización local de la biodiversidad y el desarrollo de nuevos saberes y prácticas culturales en torno a dichos recursos.

En la región de la Comarca Andina del Paralelo $42^{\circ} \mathrm{S}$ (CAP 42 , Argentina), inmersa en los bosques andino-patagónicos, los frutos silvestres son utilizados por las poblaciones rurales y urbanas, además de integrar circuitos de comercialización (J.J. Ochoa obs. personal). Sin embargo, no se han realizado estudios etnobotánicos que permitan documentar saberes y usos, actuales y pasados, de las especies silvestres nativas con frutos comestibles, así como la demanda por parte del mercado y el grado de comercialización de los mismos en esta región.

En este sentido, este trabajo se propone los objetivos de a) documentar la riqueza de especies silvestres con frutos comestibles, conocidas y utilizadas, en dos áreas de la CAP $42^{\circ}$; b) describir los principales tipos de usos y preparaciones con estos frutos; y c) aproximarnos a conocer los circuitos de comercialización de estas especies.

\section{MATERIALES Y MÉTODOS Sitio de Estudio}

Se trabajó en la Cuenca del Lago Puelo, específicamente en los parajes Río Turbio (Parque Nacional Lago Puelo, Parque Provincial y Reserva Forestal Río Turbio) y El Desemboque (provincia de Chubut, Argentina), ambos incluidos en la CAP $42^{\circ}$ (Fig. 1). Se trata de una zona de valles lacustres, con altitudes de fondo de valle de entre los $150 \mathrm{~m}$ y $250 \mathrm{~m}$. El clima es templado, con temperaturas medias invernal y estival de $6,0^{\circ} \mathrm{C}$ y $16,5^{\circ} \mathrm{C}$, respectivamente, y precipitación media anual de $1.450 \mathrm{~mm}$ con predominio en otoño-invierno. El paraje Río Turbio se encuentra dentro de dos jurisdicciones estatales: el Parque Nacional Lago Puelo (26.674 has.) y el Parque Provincial y Reserva Forestal Rio Turbio (50.000 has), conforma un área protegida desde 1937 y 1994, respectivamente. El área del paraje Río Turbio presenta dominancia del bosque mixto de coihue (Nothofagus dombeyi) y ciprés de la cordillera (Austrocedrus chilensis). En los sectores occidentales, donde las precipitaciones son más abundantes, se registra la presencia de especies arbóreas típicas de la selva valdiviana, como Persea lingue, Aextoxicon punctatum, Eucryphia cordifolia, Weinmannia trichosperma y Caldcluvia paniculata, entre otras.

Desde principio de siglo XX el paraje Río Turbio se encuentra habitado por pobladores dedicados a la ganadería y actividades agrícolas. En la actualidad lo habitan alrededor de 20 familias, con economías de subsistencias basadas en la ganadería extensiva, pequeñas producciones agrícolas, turismo y empleo estatal. El paraje conocido como El Desemboque corresponde al valle que atraviesa el Río Epuyén antes de desembocar en el Lago Puelo. Habitado por unas 30 familias, esta área corresponde originalmente a bosques mixtos, sin embargo, incendios recurrentes, sumados a las actividades productivas de ganadería y producción de fruta fina, han transformado notablemente la composición vegetal de esta zona. Sus principales actividades económicas actuales son la ganadería extensiva, cultivos de cerezas, frambuesas y otras frutas finas y, de manera creciente, actividades turísticas. 


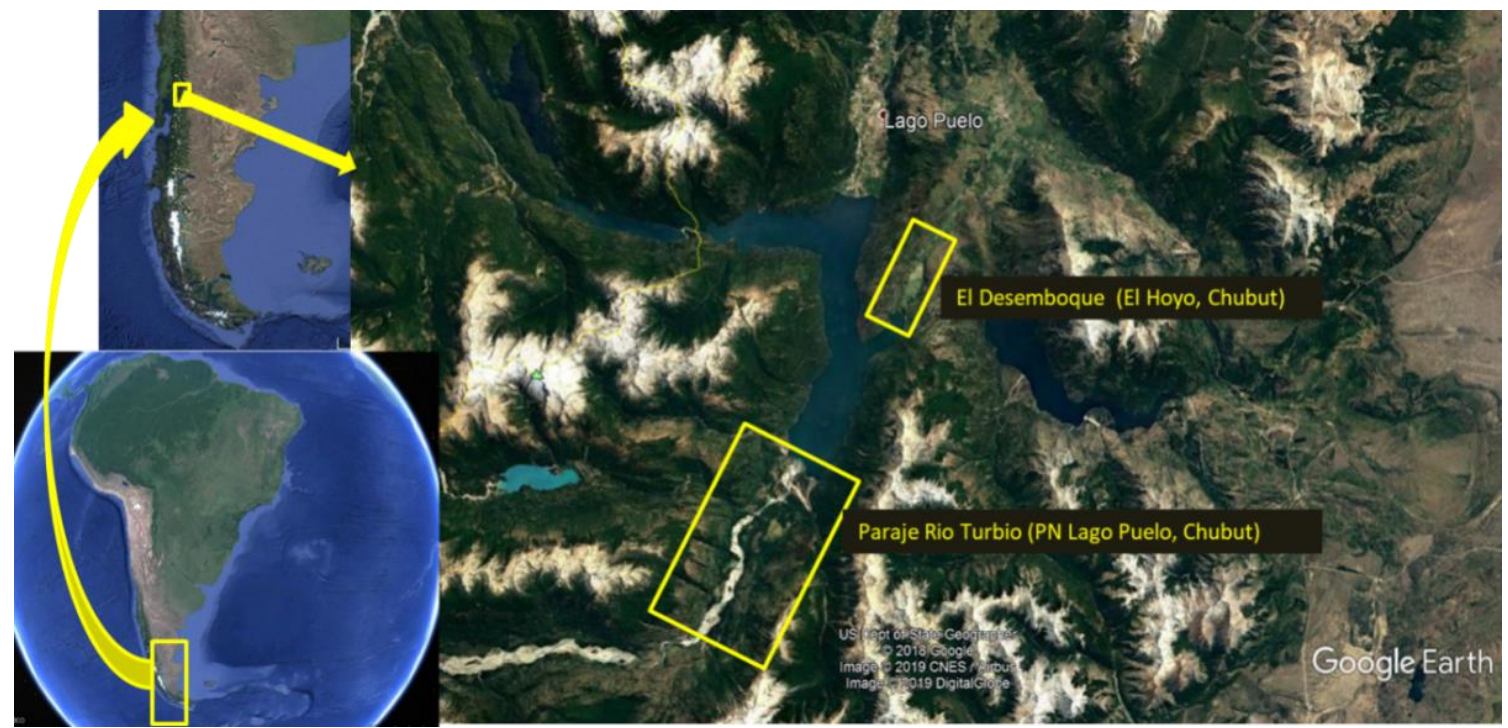

Figura 1. Sitios de estudio.

A través de entrevistas semi-estructuradas, charlas abiertas y observación participante, durante los años 2018 y 2019 se entrevistaron 22 personas, 13 hombres y 9 mujeres, de entre 40 y 98 años (promedio de 58 años). La selección de los entrevistados resulto del proceso de vinculación con referentes de cada comunidad y la aplicación de la metodología "bola de nieve" (ALBUQUERQUE et al., 2008). Previamente a la entrevista se informó sobre los objetivos de la investigación y los alcances de la misma, obteniendo el consentimiento libre informado en cada caso, siguiendo el Código de Ética de la Sociedad Internacional de Etnobiología (ISE, 2006). Las personas entrevistadas, son habitantes históricos de la región dedicados a la ganadería extensiva, actividades agrícolas, empleos estatales, actividades turísticas (cabalgatas) y otras actividades laborales informales. Las entrevistas incluyeron preguntas acerca de los frutos silvestres nativos que conocían y sus propiedades de uso. Para cada especie mencionada se consultó si ésta era consumida en la actualidad, de qué forma, dónde, cuándo y cuánto se recolecta, y si los frutos son intercambiados o comercializados. En este último caso, también se consultó acerca de las cantidades vendidas y a quién se dirigen las ventas. También se indagó en torno a los usos pasados por parte de padres y/o abuelos, y las razones de las transformaciones en los usos. Sumado a esto, se entrevistó a referentes de la comercialización de frutos, identificados tras las entrevistas a recolectores, acopiadores y referentes institucionales de la producción local. En este caso se documentó desde cuándo se realiza la actividad, los volúmenes manejados y los principales destinos de comercialización.

Para el reconocimiento del material vegetal se realizaron salidas junto a pobladores en búsqueda de colectar las especies mencionadas. Las especies fueron determinadas en laboratorio, siguiendo a Zuluoga y Morrone (1999). Todo el material vegetal recolectado fue depositado en el Herbario del Instituto de Investigaciones en Recursos Naturales, Agroecología y Desarrollo Rural (IRNAD) de la Universidad Nacional de Río Negro, asignando los siguientes números de Vouchers: Aristotelia chilensis (Molina) Stuntz/JJO 0001; Gevuina avellana Molina JJO 0002; Luma apiculata (DC.) Burret JJO 0003; Potentilla chiloensis (L.) Mabb.JJO 0004; Rubus geoides Sm. JJO 0005; Berberis microphylla G. Forst 0006; Berberis darwinii Hook. JJO 0007; Ugni molinae Turcz JJO 0008; Schinus patagonicus (Phil.) I.M. Johnst. ex Cabrera JJO 0009; Ribes magellanicum Poir JJO 0010; Amomyrtus luma (Molina) D. Legrand \& Kausel JJO 0011; Gaultheria L. sp. JJO 0012; Rubus ulmifolius Schott. JJO 0013; Sambucus nigra L. JJO 0014; Rosa rubiginosa L. JJO 0015.

\section{RESULTADOS Y DISCUSIÓN}

Al momento, documentamos una riqueza total de 15 especies, 12 nativas y 3 exóticas, conocidas por los pobladores entrevistados por sus frutos comestibles, lo que resulta un número importante si consideramos la pequeña área estudiada y las 36 especies nativas con frutos comestibles que tenemos registradas para toda la distribución de los bosques andinos de la Patagonia. Desde el punto de vista botánico, la mayor riqueza de especies nativas con frutos comestibles se presenta en la familia Mirtaceae (3); sin embargo, al incluir las especies silvestres exóticas, la familia de las Rosáceas ocupa ese lugar (4). La mayor proporción de las especies mencionadas son arbustos, que en algunos casos pueden comportarse como árboles (e.g: G. avellana, L. apiculata), mientras que sólo 
dos especies son hierbas perennes ( $P$. chiloensis y $R$. geoides). Respecto al tipo de frutos, con excepción de G. avellana, todas las especies presentan frutos carnosos, siendo las bayas las más representadas. Al observar las frecuencias de citas podemos observar que, junto a las especies exóticas (rosa mosqueta, sauco y murra), el maqui, el calafate y la murta son las especies nativas más citadas (Fig. 2). Sin embargo, al considerar si las especies son utilizadas para elaboraciones en la actualidad, podemos notar que sólo las especies exóticas muestran altos índices de uso en comparación con las plantas nativas, de las cuales solo el maqui y la murta siguen siendo utilizadas (Fig. 2.). La incorporación de plantas exóticas silvestres en las dietas y elaboraciones locales ya ha sido señalado en poblaciones suburbanas y rurales del noroeste Patagónico como un aspecto relevante de las farmacopeas y el conocimiento botánico local (LADIO, 2005; LADIO et al., 2013), dado que muchas de estas especies han sido parte de dietas mediterráneas de los inmigrantes europeos que colonizaron la Patagonia durante los siglos XIX y XX, dando forma a las heterogéneas culturas locales de la CAP $42^{\circ}$. Sumado a esto, las especies exóticas asilvestradas que se utilizan en esta región, por ejemplo, la rosa mosqueta, se comportan como invasivas (DAMASCOS y GALLOPIN, 1992) alcanzando altas densidades en áreas antropizadas y brindando una oferta de frutos muchas veces de mayor disponibilidad que las especies nativas. Finalmente, un último aspecto que favorece este mayor consenso de citas y aprovechamiento actual de las especies exóticas es el del valor económico, y su integración a circuitos de comercialización regional (QUIROGA y BLASQUIZ, 2019).

Respecto a los tipos de usos mencionados (Fig. 3), vemos que algunas especies sólo son reconocidas por sus propiedades comestibles (por ejemplo: laura, luma, michay), siete especies presentan adicionalmente usos medicinales, en ocasiones por sus frutos (ej.: maqui, avellano, zarzaparrilla) o por sus hojas (ej.: murta). Cinco especies presentan otros tipos de usos, como, por ejemplo, forrajero (avellano) y ornamental (arrayán) (Tabla 1). El reconocimiento de múltiples propiedades de una especie es un atributo que confiere posibilidades para el mantenimiento de las prácticas de uso, al brindar mayores oportunidades para la interacción. El sauco, la mosqueta y el maqui son buenos ejemplos de esto. Otros factores mencionados anteriormente (por ejemplo, la abundancia y disponibilidad de frutos) explicarían el caso de la murra, especie apreciada sólo por su propiedad comestible, pero con un alto consenso de citas y uso actual.

Al comparar los usos actuales y pasados de las especies, podemos observar que las especies nativas son menos utilizadas que en el pasado. Los entrevistados no utilizan actualmente todas las especies que mencionan conocer por sus frutos comestibles (Fig. 2), pero sí mencionaron que especies como el maqui, la zarzaparrilla o el avellano, que hoy no utilizan, fueron utilizadas por sus padres y/o abuelos en el pasado para diferentes elaboraciones. Además, quienes utilizan alguna de estas especies en el presente, mencionaron que en el pasado eran utilizadas con más frecuencia y aprovechadas de múltiples maneras, hoy no practicadas. Así, por ejemplo, el avellano no es recolectado ni consumido actualmente, aunque en el pasado, su aprovechamiento era una práctica común: sus frutos recolectados se molían y se mezclaba con harina de trigo para la elaboración de "ñaco"; o se utilizaban las semillas molidas para la preparación de una infusión similar al café. Las semillas de esta especie eran prensadas para extraer su aceite que era efectivo para el tratamiento de quemaduras. También la murta muestra alto grado de desuso, tanto por el hecho de que el $50 \%$ de los entrevistados ya no utilizan la especie, sino incluso en el hecho de que ciertas elaboraciones no son preparadas como antes, por ejemplo, el "murtado", una bebida tradicional en la que los frutos de murta se dejan macerando en alcohol destilado. Otro ejemplo de desuso es el señalado para las bayas de zarzaparrilla, que en el pasado fueron utilizados por familias para la preparación de dulces e incluso medicinalmente para tratar dolores musculares. Este desuso de las especies nativas respecto a la vigencia como a la multiplicidad, contrasta con lo que ocurre cocn las especies exóticas citadas, que presentan altos consensos y un uso vigente en la actualidad. Desde la perspectiva local, se mencionaron razones que explican este menor aprovechamiento de los frutos nativos: a) cambios culturales de acceso a otros alimentos manufacturados; y b) cambios ecológicos que hicieron menos disponibles a estas especies, por ejemplo, incendios en El Desemboque que afectaron las poblaciones de murta. En el caso del maqui, pareciera ocurrir un fenómeno opuesto, dado que quienes lo recolectan para su comercialización mencionaron que en la actualidad las recolecciones son mayores que en el pasado dado la demanda por parte de quienes compran sus frutos. 
Tabla 1: Plantas nativas y exóticas conocidas y utilizadas por pobladores de Río Turbio (PN Lago Puelo, Chubut) y El Desemboque (Chubut). Frc: frecuencia de citas de especies conocidas; Fru: frecuencia de citas para especies utilizadas en la actualidad; Tipo de fruto; Usos del fruto y otros órganos: (C) Comestible, (B): Bebida, (M) Medicinal, (T) Tintóreo, (F) Forrajero, (E) Etnoveterinario, (O) Ornamental, (Ot): otro uso; Comercialización: Si (Presenta), No (presenta).

\begin{tabular}{|c|c|c|c|c|c|}
\hline $\begin{array}{l}\text { Nombre local/ } \\
\text { Especie/Familia }\end{array}$ & Frc/Fru & Tipo de fruto & Usos del fruto & $\begin{array}{l}\text { Usos de otros } \\
\text { órganos } \\
\end{array}$ & Comercialización \\
\hline $\begin{array}{l}\text { Maquí } \\
\text { Aristotelia chilensis (Molina) } \\
\text { Stuntz } \\
\text { Elaeocarpaceae }\end{array}$ & $1 / 0,22$ & Baya & $\begin{array}{l}\text { C: crudo, } \\
\text { elaboración de } \\
\text { dulces, chicha. } \\
\text { M: fruto seco } \\
\text { en infusión }\end{array}$ & $\begin{array}{l}\text { Ot: hojas para } \\
\text { curanto }^{1} \text {. M: hojas } \\
\text { en infusión }\end{array}$ & $\mathrm{Si}$ \\
\hline $\begin{array}{l}\text { Calafate } \\
\text { Berberis microphylla } \text { G. Forst } \\
\text { Berberidaceae }\end{array}$ & $1 / 0$ & Baya & $\begin{array}{l}\text { C: crudo, } \\
\text { elaboración de } \\
\text { dulces }\end{array}$ & T: raíz & No \\
\hline $\begin{array}{l}\text { Murta } \\
\text { Ugni molinae Turcz } \\
\text { Mirtaceae }\end{array}$ & $0,9 / 0,45$ & Baya & $\begin{array}{l}\text { C: crudo, } \\
\text { elaboración de } \\
\text { dulce, bebida }\end{array}$ & $\begin{array}{l}\text { M: hojas en } \\
\text { infusión }\end{array}$ & No \\
\hline $\begin{array}{l}\text { Avellano } \\
\text { Gevuina avellana Molina } \\
\text { Proteaceae }\end{array}$ & $0,54 / 0$ & Nuez & $\begin{array}{l}\text { C: tostados, } \\
\text { molido en } \\
\text { harinas. } \mathbf{M} \text { : } \\
\text { aceite extraído } \\
\text { de las } \\
\text { semillas. }\end{array}$ & $\mathbf{F}$ & No \\
\hline $\begin{array}{l}\text { Michay } \\
\text { Berberis darwinii Hook } \\
\text { Berberidaceae }\end{array}$ & $0,45 / 0$ & Baya & C: crudo & No & No \\
\hline $\begin{array}{l}\text { Frutilla silvestre } \\
\text { Potentilla chiloensis (L.) } \\
\text { Mabb. } \\
\text { Rosaceae }\end{array}$ & $0,31 / 0$ & Conocarpo & C: crudo & $\begin{array}{l}\text { E: hojas en } \\
\text { infusión }\end{array}$ & No \\
\hline $\begin{array}{l}\text { Laura } \\
\text { Schinus patagonicus (Phil.) } \\
\text { I.M. Johnst. ex Cabrera } \\
\text { Anacardiaceae }\end{array}$ & $0,22 / 0$ & Drupa & C: crudo & No & No \\
\hline $\begin{array}{l}\text { Zarzaparrilla } \\
\text { Ribes magellanicum Poir } \\
\text { Grossulariaceae }\end{array}$ & $0,22 / 0$ & Baya & C: crudo & No & No \\
\hline $\begin{array}{l}\text { Chaura } \\
\text { Gaultheria sp. L. Ericaceae }\end{array}$ & $0,22 / 0$ & Baya & $\begin{array}{l}\text { C: crudo, } \\
\text { elaboración de } \\
\text { dulces }\end{array}$ & No & No \\
\hline $\begin{array}{l}\text { Arrayán } \\
\text { Luma apiculata (DC.) Burret } \\
\text { Mirtaceae }\end{array}$ & $0,18 / 0-$ & Baya & $\begin{array}{l}\text { C: crudo, } \\
\text { elaboración de } \\
\text { dulce }\end{array}$ & $\begin{array}{l}\text { M: hojas en } \\
\text { infusión. O: toda } \\
\text { la planta }\end{array}$ & No \\
\hline $\begin{array}{l}\text { Luma } \\
\text { Amomyrtus luma (Molina) D. } \\
\text { Legrand \& Kausel } \\
\text { Mirtaceae }\end{array}$ & $0,09 / 0$ & Baya & C: crudo & No & No \\
\hline $\begin{array}{l}\text { Frutilla de lenga } \\
\text { Rubus geoides } \mathrm{Sm} \\
\text { Rosaceae }\end{array}$ & $0,09 / 0$ & Polidrupa & C: crudo & No & No \\
\hline $\begin{array}{l}\text { Murra } \\
\text { Rubus ulmifolius Schott } \\
\text { Rosaceae }\end{array}$ & $1 / 1$ & $\begin{array}{l}\text { Frutos silve } \\
\text { Polidrupa }\end{array}$ & $\begin{array}{l}\text { tres exóticos } \\
\text { C: crudo, } \\
\text { elaboración de } \\
\text { dulces }\end{array}$ & No & $\mathrm{Si}$ \\
\hline $\begin{array}{l}\text { Rosa mosqueta } \\
\text { Rosa rubiginosa } \mathrm{L} . \\
\text { Rosaceae }\end{array}$ & $1 / 1$ & Cinorrodón & $\begin{array}{l}\text { C: elaboración } \\
\text { de dulces. } \\
\text { Medicinal: } \\
\text { infusiones }\end{array}$ & No & $\mathrm{Si}$ \\
\hline $\begin{array}{l}\text { Sauco } \\
\text { Sambucus nigra L. } \\
\text { Adoxaceae }\end{array}$ & $1 / 0,63$ & Baya & $\begin{array}{l}\text { C: crudo, } \\
\text { elaboración de } \\
\text { dulces. } \mathbf{M} \text { : } \\
\text { preparación de } \\
\text { jarabes }\end{array}$ & B: inflorescencia & $\mathrm{Si}$ \\
\hline
\end{tabular}




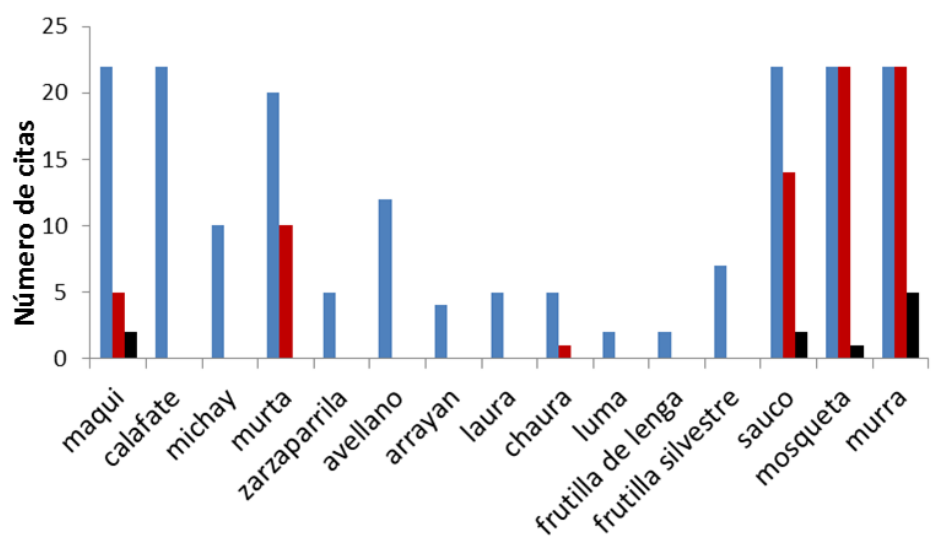

Fig. 2. Frecuencia de citas de identificación, uso actual y número de personas que comercializan las especies silvestres con frutos comestibles por los pobladores de Río Turbio y El Desemboque.

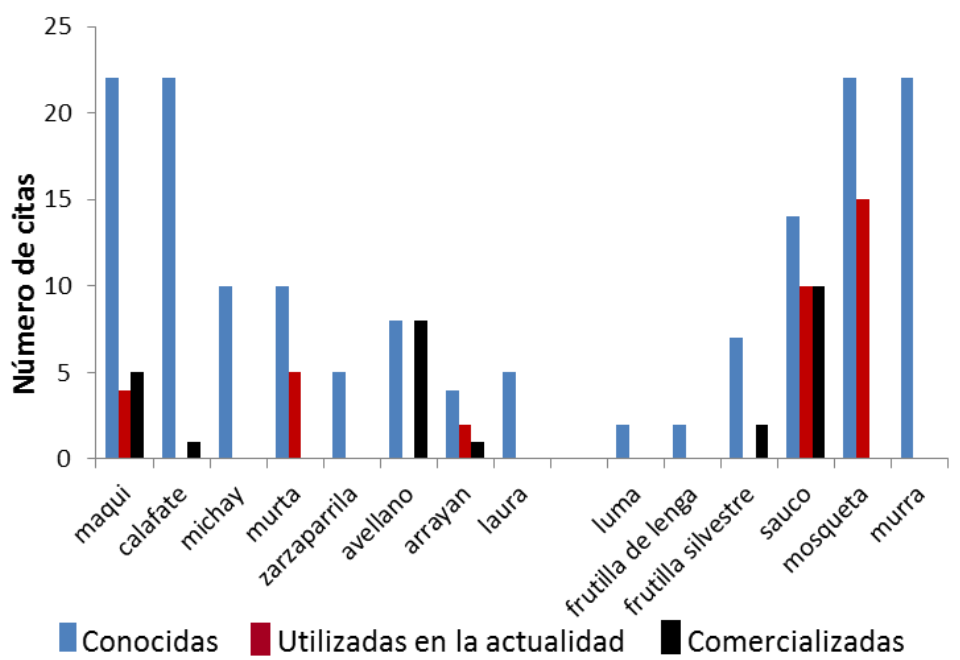

Figura 3. Multiplicidad de usos mencionados por los pobladores de Río Turbio y El Desemboque.

Finalmente, al analizar la comercialización, podemos observar que sólo una especie nativa, el maqui, es recolectada actualmente para su comercialización por dos de los pobladores entrevistados, mientras que las tres especies exóticas son comercializadas, aunque también por un número bajo de entrevistados (Fig. 2). La murta fue señalada como un fruto antaño comercializado, algo que no ocurre en la actualidad. Respecto a la antigüedad y frecuencia de esta práctica de recolección y comercialización de estos frutos podemos señalar que todos los pobladores entrevistados mencionan que la comercialización de los frutos nativos (maqui y murta) comenzó en los años 80, como una demanda de dulcerías de Bariloche, Gaiman y El Bolsón. Respecto a la murta, los pobladores que participaban de dicha actividad recuerdan la cosecha de unos $200 \mathrm{~kg}$ o incluso más por temporada y que fue alrededor de los años 2000 cuando se la dejó de comercializar. En el caso del maqui, la demanda de estas dulcerías fue más tardía (años noventa), con valores similares de recolección. Si bien se reconoce que el calafate es una especie comercializada, ésta es recolectada fuera del área de estudio para su venta, y ninguno de los entrevistados realiza esta actividad. En la actualidad, los principales compradores de maqui son una dulcería de Bariloche, una heladería regional y un exportador nacional.

Los diferentes eslabones en la cadena de comercialización asociados al maqui son: la recolección (pobladores rurales), acopio (poblador local), transporte (servicio tercerizado o realizado por el propio comerciante); elaboración - comercialización (dulcerías, heladerías, empresa dedicada al mercado nacional e internacional del producto liofilizado) (Fig. 4). Si bien en esta primera aproximación pudimos identificar sólo a dos pobladores que recolectan maqui con fines comerciales, las entrevistas con acopiadores y empresas dedicadas a la elaboración de productos a partir de esta especie, y trabajo de campo en curso dentro de otras poblaciones de la CAP $42^{\circ}$, dan cuenta de una red más extensa de recolectores. Estos recolectores venden los frutos cosechados en poblaciones silvestres durante los meses de Febrero - Marzo, directamente a tres destinatarios: a) una dulcería de Bariloche 
que retira la producción en las casa de los productores; b) a un acopiador de la localidad de El Hoyo, que luego re-vende a dulcerías y a una heladería local; y c) a una empresa nacional que traslada la producción a la ciudad de Buenos Aires, donde se la liofiliza y empaqueta para su venta en mercados nacional e internacional. Los valores de venta de fruto rondan los 40 - 70 pesos por $\mathrm{kg}$. Las entrevistas con el acopiador mencionado, y con referentes de la empresa que comercializa a través de la exportación nos permiten señalar que la compra a la red de recolectores de la CAP $42^{\circ}$ oscila alrededor de los $2.000 \mathrm{Kg}$ por temporada, y que esta producción no llega a cubrir las demandas.

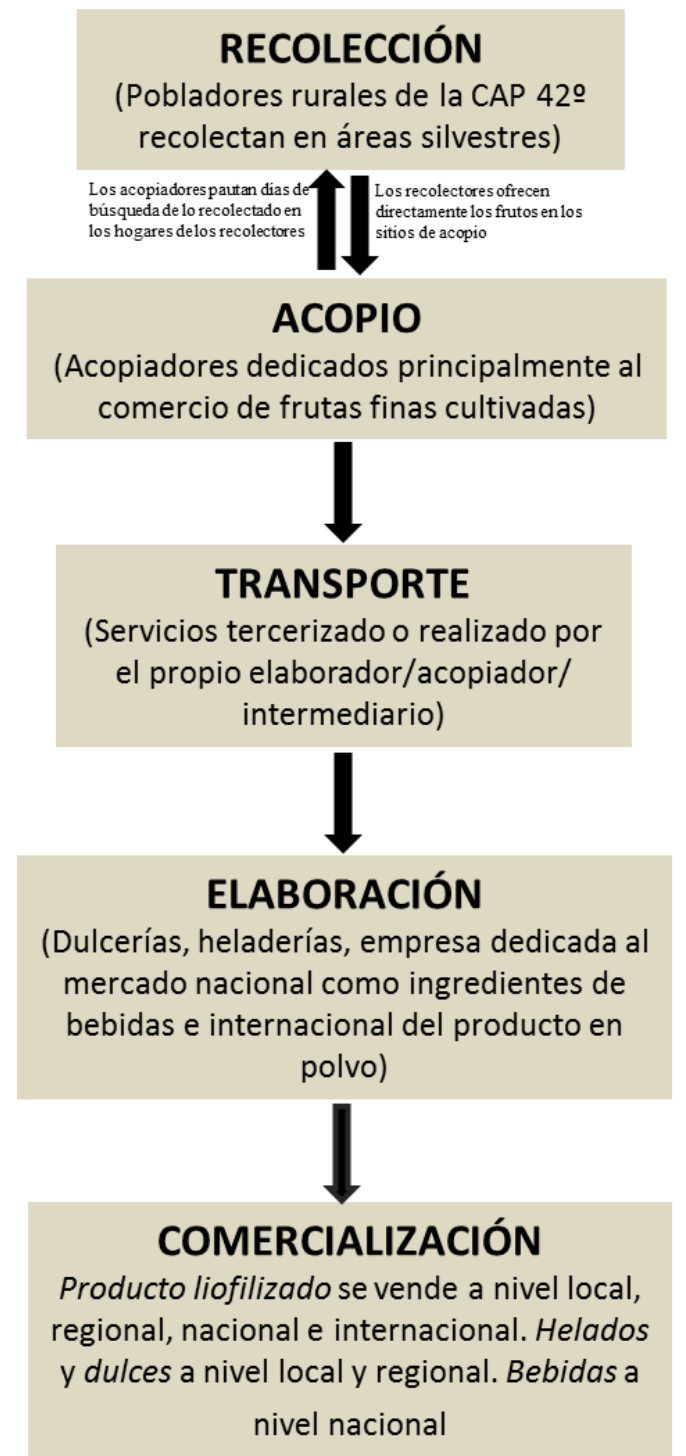

Fig. 4. Esquema de los eslabones y actores involucrados en el circuito de recolección- comercialización de Aristotelia chilensis (Molina) Stuntz en la CAP $42^{\circ}$, Argentina.

Además, estos actores señalaron que las principales dificultades de la cadena de comercialización es la logística para acopiar la producción, dado que los recolectores trabajan de manera individual, lo que implica coordinación para retirar los frutos en diferentes puntos geográficos. También se señaló la falta de uniformidad en la producción que se entrega, que muchas veces se encuentra mezclada con hojas y tallos. Los frutos de maqui requieren refrigeración tras la cosecha, y dado que muchas veces el recolector no cuenta con las herramientas para la correcta manipulación, la degradación de los mismos es un punto crítico en esta cadena, ya que esto podría afectar el contenido de compuestos antioxidantes, una de las principales razones para la creciente demanda del mercado. Por último, en el caso de la empresa que comercializa polvo liofilizado de maqui, la ausencia de un liofilizador en la CAP $42^{\circ}$ fue señalada como un factor que dificulta, y encarece, el proceso de manipulación de los frutos hasta la obtención del producto final.

En Chile, el maqui y otras especies nativas han recibido mayor atención por parte de instituciones del Estado (TACÓN CLAVAÍN, 2004; VALDEBENITO et al., 2015), que caracterizan e 
impulsan al aprovechamiento de estos recursos. A modo de referencia, distintos estudios realizados en Chile indican que: a) existe una fuerte explotación de áreas silvestres que estaría afectando negativamente la dinámica poblacional del maqui (ARRIBILLAGA y ZEGERS, 1998); b) la recolección es realizada principalmente por pobladores rurales e indígenas (organizados en cooperativas o individuales) (CORTÉZ et al., 2017); c) entre los años 2009 y 2015 las diez principales empresas exportaron un promedio de $535.000 \mathrm{~kg}$ de fruto lo que representó ventas por 6,5 millones de dólares (MUÑOZ, 2018); y d) se están desarrollando procesos de domesticación de esta especie (VOGET et al., 2016) y un importante número de huertos productivos (NAVARRO ANTÚNEZ, 2014). En Argentina aún la importancia económica de este recurso y la búsqueda de sustentabilidad para el uso del mismo están subestimadas; existen vacíos de información respecto a los eslabones, actores involucrados en la comercialización y volúmenes y destinos de exportación.

\section{CONCLUSIONES}

El uso de frutos silvestres de plantas nativas comestibles en las poblaciones de la CAP $42^{\circ}$ en las que se trabajó muestra estar en proceso de abandono, tanto a nivel de la riqueza de especies como en cuanto a la multiplicidad de usos actuales. Como en otras regiones del mundo, las razones que explican dicho proceso de abandono se asocian a la creciente adopción de prácticas alimenticias y dependencia en productos del mercado, así como a las transformaciones ecológicas que experimentan estos paisajes (por ejemplo incendios, cambios del clima) y que afectan la disponibilidad de estos recursos.

La demanda creciente del mercado sobre los frutos silvestres, particularmente el maqui, Aristotelia chilensis (Molina) Stuntz, la única especie nativa comercializada en la actualidad en base a su alto contenido de antioxidantes (FREDES, 2009), sin duda impulsará a que cada vez más pobladores se involucren en la recolección. Esto activará, en la práctica, diversidad de manejos, generando mayor presión e incierto futuro sobre las poblaciones de esta especie. Este trabajo aporta los primeros datos sobre la etnobotánica y actores involucrados en la comercialización de frutos comestibles silvestres en la región andino-patagónica de Argentina. Sin embargo, es necesario llevar adelante investigaciones que profundicen las evaluaciones etnobotánicas en diferentes áreas de la CAP $42^{\circ}$. También son necesarios estudios ecológicos para evaluar el impacto de la recolección en poblaciones silvestres de las especies nativas, particularmente A. chilensis, así como ensayos de selección y cultivo de las especies nativas valoradas y demandadas por el mercado.

\section{AGRADECIMIENTOS}

A los pobladores de El Desemboque y Río Turbio (PN Lago Puelo) por compartir sus saberes y tiempos en esta investigación, así como a los referentes de la comercialización (acopiadores y empresarios) que nos brindaron datos y perspectivas sobre la comercialización de frutos nativos. Al personal del PN Lago Puelo por facilitarnos acceso al Río Turbio. A los dos evaluadores anónimos que colaboraron a mejorar la redacción de este trabajo. Esta investigación fue financiada por la Universidad Nacional de Río Negro a través del PI UNRN 40-B-663 “Comportamiento productivo, arquitectura y conocimientos ecológicos locales de plantas nativas con frutos comestibles de la Comarca Andina del Paralelo 42 S, Provincias de Río Negro y Chubut, Argentina"

\section{BIBLIOGRAFÍA}

ALBUQUERQUE UP, et al. Métodos e técnicas para a coleta de dados. En ALBUQUERQUE UP, LUCENA RFP, CUNHA LVFC (eds). Métodos e Técnicas na Pesquisa Etnobotânica. Recife, Pernambuco, Brazil: Núcleo de Publicações em Ecologia e Etnobotânica Aplicada (NUPEEA), 2008, 37-62.

ARRIBILlAGA D, ZEGERS MT. Explotación Industrial del Calafate. Tierra Adentro, Chile, 21, 18-19, julio-agosto de 1998 .

BERNAL V, et al. Role of wild plant foods among late Holocene hunter-gatherers from Central and North Patagonia (South America): An approach from dental evidence. American Journal of Physical Anthropology, New York, 133(4), 1047-1059. 2007.

BHARUCHA Z, PRETTY J. The roles and values of wild foods in agricultural systems. Philosophical Transactions of the Royal Society B: Biological Sciences, London 3651, 2913-2926. 2010.

CIAMPAGNA LM, CAPARELLI AC. Historia del uso de las plantas por parte de las poblaciones que habitaron la Patagonia Continental Argentina. Revista Arqueología, Buenos Aíres, 6, 45-75. 2012.

CORTÉS M, et al. La recolección de productos forestales no madereros por mujeres campesinas del sur de Chile: reconfigurando la tensión entre lo local y lo global. Revista Iberoamericana de Viticultura, Agroindustria y Ruralidad, Santiago de Chile, 4(12), 22-45. 2017. 
DAMASCOS MA, GALLOPIN GG, Ecología de un arbusto introducido (Rosa rubiginosa L. Rosa eglanteria L.): riesgo de invasión y efectos en las comunidades vegetales de la región andino-patagónica de Argentina. Revista Chilena de Historia Natural, Santiago de Chile, 65, 395-407. 1992.

FREDES C. Antioxidantes en berries nativos chilenos. Boletín Latinoamericano y del Caribe de plantas medicinales y aromáticas, Santiago de Chile, 8(6), 469-478. 2009.

ISE. International Society of Ethnobiology. Code of Ethics. 2006 Disponible en:http://ethnobiology.net/code-of-ethics. Fecha de acceso: 10 de Junio 2018.

LADIO AH. The maintenance of wild edible plant gathering in a Mapuche community of Patagonia. Economic Botany, New York, 55(2), 243-254. 2001.

LADIO AH. Malezas exóticas comestibles y medicinales utilizadas en poblaciones del Noroeste patagónico: aspectos etnobotánicos y ecológicos. Boletín Latinoamericano y del Caribe de Plantas Medicinales y Aromáticas, Santiago de Chile, 4(4), 11-17. 2005.

LADIO AH, et al. Etnobotánica aplicada en Patagonia: la comercialización de malezas de uso comestible y medicinal en una feria urbana de San Carlos de Bariloche (Río Negro, Argentina). Boletín Latinoamericano y del Caribe de Plantas Medicinales y Aromáticas, Santiago de Chile, 12(1). 2013.

MUÑOZ RR. Perspectiva del Mercado Internacional para el desarrollo de la industria del maqui: Un análisis de las empresas en Chile.. Serie de estudios para la innovación FIA. Primera edición. Santiago de Chile. Fundación para la Innovación Agraria, 2015, pp 70.

NAVARRO ANTÚNEZ CA. Estados fenológicos y componentes del rendimiento en clones de maqui (Aristotelia chilensis) establecidos en dos localidades de la región del Maule. 2014. pp: 45, Disertación Doctoral, Escuela de Agronomía, Universidad de Talca, Talca, Chile.

OCHOA JJ, et al. Uso de recursos herbolarios entre mapuches y criollos de la comunidad campesina de Arroyo Las Minas (Río Negro, Patagonia Argentina). Boletín Latinoamericano y del Caribe de Plantas Medicinales y Aromáticas, Santiago de Chile, 9(4), 269-275. 2010.

PIMENTEL D, et al. The value of forests to world food security. Human ecology, New York, 25(1), 91-120. 1997.

TACÓN et al. El mercado de los PFNM y su papel en la conservación de la ecorregión de los bosques valdivianos. Primera edición. Valdivia, Chile. WWF-Red PFNM de Chile. 2004. 134 pp.

VALDEBENITO G, et al. Modelos de negocios sustentables de recolección, procesamiento y comercialización de Productos Forestales No Madereros (PFNM) en Chile. Serie de estudios para la innovación FIA. Primera edición. Santiago de Chile. Fundación para la Innovación Agraria, 2015, pp 22

VOGEL H, et al. Domestication and sustainable production of wild crafted plants with special reference to the Chilean maqui berry (Aristotelia chilensis). Julius-Kühn-Archiv, Quedlinburg, 453, 50-52. 2016.

ZULOAGA FO, MORRONE O. Catálogo de las plantas vasculares de la República Argentina. II. Monographs in Systematic Botany, Missouri Botanical Garden. Disponible en: http://www.darwinion.gov.ar. 1999. Consultado en mayo de 2019. 